\title{
Correction to: Nodal set of strongly competition systems with fractional Laplacian
}

Shan Zhang and Zuhan Liu

Correction to: Nonlinear Differ. Equ. Appl. 22 (2015), 1483-1513 https://doi.org/10.1007/s00030-015-0332-3

The authors would like to report a mistake in the proof of Theorem 4.1 in [3]. Theorem 4.1 is related to the proof of an Almgren monotonicity formula, which is the starting point and core of the original paper. However, we find that this formula only holds in the case of two densities. Moreover, the estimate on the Hausdorff dimension of the singular set was incorrect. The following is the corrected formulation and proof. We apologize for any inconvenience caused.

Suppose that $M=2$, and let $w(x, y)=v_{1}(x, y)-v_{2}(x, y)$. Then $w$ satisfies

$$
\begin{cases}\triangle w=0 & \text { in } \mathbb{R}_{+}^{n+1}, \\ \partial_{\nu} w=0 & \text { on } \Omega \times\{0\} .\end{cases}
$$

Moreover, by Theorem 2.1 in [3] $w$ is locally $C^{0, \alpha}$ continuous in $\mathbb{R}_{+}^{n+1} \cup \Omega$. Then by the segregation result in Theorem 2.1 , the nodal set $\Gamma(v(\cdot, 0))$ is exactly the zero set of $w(\cdot, 0)$, and we denote it by $\Gamma(w(\cdot, 0))$.

We define for every $x_{0} \in \Omega$ and $r \in\left(0, \operatorname{dist}\left(x_{0}, \partial \Omega\right)\right)$,

$$
\begin{aligned}
& E(r)=E\left(\left(x_{0}, 0\right), w, r\right)=\frac{1}{r^{n-1}} \int_{B_{r}^{+}\left(x_{0}, 0\right)}|\nabla w|^{2} d x d y, \\
& H(r)=H\left(\left(x_{0}, 0\right), w, r\right)=\frac{1}{r^{n}} \int_{\partial^{+} B_{r}\left(x_{0}, 0\right)} w^{2} d \sigma
\end{aligned}
$$

The original article can be found online at https://doi.org/10.1007/s00030-015-0332-3. 
and the Almgren's frequency

$$
N\left(\left(x_{0}, 0\right), w, r\right)=\frac{E\left(\left(x_{0}, 0\right), w, r\right)}{E\left(\left(x_{0}, 0\right), w, r\right)} .
$$

Let $x_{0}$ be fixed. Since $w \in H_{l o c}^{1}$, both $E$ and $H$ are absolutely continuous functions on $r$. We now state the Almgren monotonicity formula, which is the corrected version of Theorem 4.1 in [3].

Theorem 0.1. Let $\widetilde{\Omega} \Subset \Omega$. For every $x_{0} \in \widetilde{\Omega}$, the Almgren frequency function $N\left(\left(x_{0}, 0\right), w, r\right)$ is nondecreasing for $r \in\left(0, \operatorname{dist}\left(x_{0}, \partial \Omega\right)\right)$, and the limit $N\left(\left(x_{0}, 0,\right) w, 0^{+}\right):=\lim _{r \rightarrow 0^{+}} N\left(\left(x_{0}, 0\right), w, r\right)$ exists and is finite. Moreover,

$$
\frac{d}{d r} \log H(r)=\frac{2 N(r)}{r} .
$$

Proof. Note that by considering an even extension $\widetilde{w}$ of $w$ to the whole $\mathbb{R}^{n+1}$, we have $\triangle \widetilde{w}=0$ in $\Omega \times \mathbb{R}$. Moreover, the following identity holds:

$$
\begin{aligned}
\int_{B_{r}\left(x_{0}, 0\right)}|\nabla \widetilde{w}|^{2} d x d y & =2 \int_{B_{r}^{+}\left(x_{0}, 0\right)}|\nabla w|^{2} d x d y, \int_{\partial B_{r}\left(x_{0}, 0\right)} w^{2} d \sigma \\
& =2 \int_{\partial^{+} B_{r}\left(x_{0}, 0\right)} w^{2} d \sigma .
\end{aligned}
$$

Hence, the rest of the proof is classical, which follows as a direct result of Theorem 2.2.3 in [1, Chapter 2].

Remark 0.2. We remark that when $M=2$, if we let $v=u_{1}-u_{2}$, then we are dealing with the nodal set of a solution to a single equation $(-\triangle)^{1 / 2} v=0$ in $\Omega$. As a consequence, Section 3 in the paper [3] is no longer needed, since we do not need a cleanup lemma in the current situation.

Remark 0.3. By using the well-known local extension realization of the fractional Laplacian and an even refection, the structure of the zero set $\{\widetilde{w}=0\}$ is very well known and follows directly from the fact the harmonic functions are real analytic. See, for instance, the book "Nodal Sets of Solutions of Elliptic Differential Equations" by Han and Lin [1], where monotonicity formulas and the full structure of the zero set are deduced. However, in the current situation, the Hausdorff dimension estimates of the nodal and singular sets of $w(\cdot, 0)$ are still unclear, and we will present some results concerning this problem.

With the Almgren monotonicity formula in hand, the regularity of the nodal set $\Gamma(w(\cdot, 0))$ follows in a standard way. To begin with, we make a blowup to obtain the possible limits of Almgren's frequency. Let $\widetilde{\Omega} \Subset \Omega$, and take some sequence $x_{k} \in \widetilde{\Omega}, t_{k} \downarrow 0$. We define a blowup sequence by

$$
w_{k}(x, y)=\frac{w\left(x_{k}+t_{k} x, t_{k} y\right)}{\rho_{k}} \text { for } x \in \Omega_{k}:=\frac{\Omega-x_{k}}{t_{k}} \text { and } y>0
$$


with

$$
\rho_{k}^{2}=\left\|w\left(x_{k}+t_{k} \cdot t_{k} \cdot\right)\right\|_{L^{2}\left(\partial^{+} B_{1}(0)\right)}^{2}=\frac{1}{t_{k}^{n}} \int_{\partial^{+} B_{t_{k}}\left(x_{k}, 0\right)} w^{2} d \sigma=H\left(\left(x_{k}, 0\right), w, t_{k}\right) .
$$

We observe that $\left\|w_{k}\right\|_{L^{2}\left(\partial^{+} B_{1}(0)\right)}=1$ and $w_{k}$ solves the system

$$
\begin{cases}-\triangle w_{k}=0 & \text { in } \mathbb{R}_{+}^{n+1} \\ \partial_{\nu} w_{k}=0 & \text { in } \Omega_{k} \times\{0\}\end{cases}
$$

In this setting, for any $z_{0} \in \Omega_{k}$ and $r \in\left(0, \operatorname{dist}\left(z_{0}, \partial \Omega_{k}\right)\right)$, the following identities hold:

$$
\begin{aligned}
& E\left(\left(z_{0}, 0\right), w_{k}, r\right)=\frac{1}{\rho_{k}^{2}} E\left(\left(x_{k}+t_{k} z_{0}, 0\right), w, t_{k} r\right), \\
& H\left(\left(z_{0}, 0\right), w_{k}, r\right)=\frac{1}{\rho_{k}^{2}} H\left(\left(x_{k}+t_{k} z_{0}, 0\right), w, t_{k} r\right),
\end{aligned}
$$

and hence

$$
N\left(\left(z_{0}, 0\right), w_{k}, r\right)=N\left(\left(x_{k}+t_{k} z_{0}, 0\right), w, t_{k} r\right) .
$$

We observe that $\left(\Omega_{k}-x_{k}\right) / t_{k} \rightarrow \mathbb{R}^{n}$ as $k \rightarrow+\infty$ because $\operatorname{dist}\left(x_{k}, \partial \Omega\right) \geqslant$ $\operatorname{dist}(\widetilde{\Omega}, \partial \Omega)>0$ for every $k$. Now, we have the following convergence result and its main consequence.

Theorem 0.4. Under previous notations, there exists $\bar{w} \in C_{\text {loc }}^{m}\left(\overline{\mathbb{R}_{+}^{n+1}}\right)$ such that, up to a subsequence, $w_{k} \rightarrow \bar{w}$ in $C_{\text {loc }}^{m}\left(\overline{\mathbb{R}_{+}^{n+1}}\right)$ for every $m \geqslant 1$. Moreover, the limit $\bar{w}$ solves

$$
\begin{cases}-\triangle \bar{w}=0 & \text { in } \mathbb{R}_{+}^{n+1}, \\ \partial_{\nu} \bar{w}=0 & \text { in } \mathbb{R}^{n} \times\{0\} .\end{cases}
$$

Corollary 0.5. Under the previous notations, suppose that one of these situations occurs:

1. $x_{k}=x_{0}$ for every $k$,

2. $x_{k} \in \Gamma(w(\cdot, 0))$ and $x_{k} \rightarrow x_{0} \in \Gamma(w(\cdot, 0))$ with $\left.N\left(x_{0}, 0\right), w, 0^{+}\right)=1$.

Then $N(0, \bar{w}, r)=N\left(\left(x_{0}, 0\right), w, 0^{+}\right)=: \alpha \geqslant 1$ for every $r>0$, and $\bar{w}=r^{\alpha} G(\theta)$, where $(r, \theta)$ are the generalized polar coordinates centered at the origin.

We now define the regular and singular parts of $\Gamma(w(\cdot, 0))$, respectively, by

$$
\begin{aligned}
& \Sigma(w(\cdot, 0)):=\left\{x \in \Gamma(w(\cdot, 0)): N\left((x, 0), w, 0^{+}\right)=1\right\}, \\
& S(w(\cdot, 0)):=\left\{x \in \Gamma(w(\cdot, 0)): N\left((x, 0), w, 0^{+}\right)>1\right\} .
\end{aligned}
$$

Then, Proposition 6.1 in [3] holds. In fact, let $\widetilde{w}$ be the even extension of $w$ to the whole $\mathbb{R}^{n+1}$, then $\Sigma(w(\cdot, 0))=\Sigma(\widetilde{w}(\cdot, 0))$. Moreover, on the regularity points $\left(x_{0}, 0\right)$, we have $\nabla \widetilde{w}\left(x_{0}, 0\right) \neq 0$ and $\partial_{y} \tilde{w}\left(x_{0}, 0\right)=0$; hence, $\Sigma$ is indeed a smooth hypersurface (of $\mathbb{R}^{n}$ ) by the implicit function theorem. 
We are left to prove the Hausdorff dimension estimates for the nodal and singular sets. To begin with, we show that Hausdorff dimension of the nodal set is not greater than $n-1$. By appropriate translation and scaling, it suffices to show that:

Proposition 0.6. (Proposition $6.2[3])$ Assume that $\partial^{0} B_{2}(0) \Subset \Omega \times\{0\}$. Then,

$$
\mathcal{H}_{\text {dim }}\left(\Gamma(w(\cdot, 0)) \cap \partial^{0} B_{1}(0)\right) \leqslant n-1 .
$$

Proof. The idea is to apply a version of the so-called Federer's Reduction Principle. We remark that the version that will be used is the one in $[3$, Theorem 6.1] with $M=1$, and $n+1$ instead of $n$.

Define the following class of functions:

$$
\mathcal{F}=\left\{w \in L_{l o c}^{\infty}\left(\overline{\mathbb{R}_{+}^{n+1}}\right): w \not \equiv 0 \text { in } \Omega \text { and satisfies }(0.1)\right\} .
$$

Let us start by checking $(A 1)$ and $(A 2)$. Hypothesis $(A 1)$ is immediately satisfied by (0.3). Moreover, let $\left|\left(x_{0}, 0\right)\right|<1, t_{k} \downarrow 0$ and $w \in \mathcal{F}$, and choose $\rho_{k}=\left\|w\left(x_{0}+t_{k} x, t_{k} y\right)\right\|_{L^{2}\left(\partial+B_{1}(0)\right)}$. Theorem 0.4 and Corollary 0.5 (Case 1) yield the existence of $\bar{w} \in \mathcal{F}$ such that (up to a subsequence) $w_{k} \rightarrow \bar{w}$ in $\mathcal{F}$ and $\bar{w}$ is a homogeneous function of degree $\alpha=N\left(\left(x_{0}, 0\right), w, 0^{+}\right) \geqslant 1$. Hence, also $(A 2)$ holds. Next, we choose the map $\mathfrak{S}$ according to different situations.

Proof of (0.8). Define

$$
\mathfrak{S}(w):=\left\{(x, 0) \in \mathbb{R}^{n} \times \mathbb{R}: w(x, 0)=0\right\},
$$

that is $\mathfrak{S}(w)=\Gamma(w(\cdot, 0)) \times\{0\}$. Note that

$$
\operatorname{dim}_{\mathcal{H}}\left(\mathfrak{S}(w) \cap B_{1}(0)\right)=\operatorname{dim}_{\mathcal{H}}\left(\Gamma(w(\cdot, 0)) \cap \partial^{0} B_{1}(0)\right) .
$$

Note also that $\mathfrak{S}(w) \cap B_{1}(0)$ is obviously closed in $B_{1}(0)$ by the continuity of $w$. It is quite straightforward to check hypothesis $(A 3)-(i)$, and the local uniform convergence considered in $\mathcal{F}$ clearly yields $(A 3)-(i i)$. By Federer's Reduction Principle, there exists $0 \leqslant d \leqslant n+1$ such that $\operatorname{dim}_{\mathcal{H}}(\mathfrak{S}(w) \cap$ $\left.B_{1}(0)\right) \leqslant d$ for any $w \in \mathcal{F}$. Moreover, there exist a $w \in \mathcal{F}$ and a linear subspace $L$ of $\mathbb{R}^{n} \times \mathbb{R}$ satisfying $\operatorname{dim}_{\mathcal{H}} L=d$ and $\mathfrak{S}(w)=L$. We need to show $d \leqslant n-1$. Since $L=\mathfrak{S}(w) \subset \mathbb{R}^{n} \times\{0\}$, we must have $d \leqslant n$. If $d=n$, then the linear subspace has to coincide with $\mathbb{R}^{n} \times\{0\}$, which means $w(\cdot, 0) \equiv 0$. This contradicts our assumption on $w$; thus, $d \leqslant n-1$.

Remark 0.7. We remark that the Hausdorff dimension of the singular set is different from that of the classical Laplace diffusion case. The anonymous referees gave a counterexample, from which we could no longer expect that the Hausdorff dimension of the singular set is at most $n-2$. Precisely, let $w: \mathbb{R}^{3} \rightarrow \mathbb{R}$ be defined as

$$
w\left(x_{1}, x_{2}, y\right)=x_{1}^{2}-y^{2} .
$$

Observe that $n=2$ in this case. Then, 


$$
\begin{cases}\triangle w=0 & \text { in } \mathbb{R}^{3}, \\ \partial_{\nu} w=0 & \text { in } \mathbb{R}^{2} \times\{0\} .\end{cases}
$$

Moreover, $w$ is homogeneous of degree 2 with respect to any point of the 1-dimensional linear subspace

$$
L=\left\{\left(x_{1}, x_{2}, y\right) \in \mathbb{R}^{3}: x_{1}=y=0\right\} \subset \mathbb{R}^{2} \times\{0\} .
$$

Thus, the singular set of this function has dimension $d=1=n-1$.

For the singular set, based on the Almgren's stratification theorem and the fact that the even extension of $w(\tilde{w})$ is harmonic in $\mathbb{R}^{n+1}$, we can give a more accurate result. Precisely, let $V$ be the set of all points $y \in \mathbb{R}^{n}$ such that $N\left((y, 0), \bar{w}, 0^{+}\right)=N\left(0, \bar{w}, 0^{+}\right)$. Now for each $j=0,1, \ldots, n-1$ we define

$$
S_{j}:=\{\xi \in S(w(\cdot, 0)): \operatorname{dim} V \leqslant j \text { for all tangent maps } \bar{w} \text { of } w \text { at }(\xi, 0)\} .
$$

We can now state the main result concerning the singular set, which is a version of Almgren's stratification theorem. Its proof follows as a direct result of Lemma 1 in [2, Section 3.4].

Theorem 0.8. Let $S_{j}, j=0,1, \ldots, n-1$ be defined as in (0.9), then we can write

$$
S_{0} \subset S_{1} \subset \cdots \subset S_{n-1}=S(w(\cdot, 0))
$$

such that the Hausdorff dimension of $S_{j} \leqslant j$ for $j=0,1, \ldots, n-1$.

\section{Acknowledgements}

The authors would like to express their sincere thanks to the anonymous referees for giving the counterexample presented in Remark 0.7, and to both the Editor and the anonymous referees for their valuable comments, suggestions and insightful questions that helped improve the content and structure of this erratum.

\section{References}

[1] Han, Q., Lin, F.H.: Nodal Sets of Solutions of Elliptic Differential Equations, books available on Hans homepage

[2] Simon, L.: Theorems on Regularity and Singularity of Energy Minimizing Maps. Based on Lecture Notes by Norbert Hungerbühler. Lectures in Mathematics ETH Zürich. Birkhäuser, Basel (1996)

[3] Zhang, S., Liu, Z.: Nodal set of strongly competition systems with fractional Laplacian. Nonlinear Differ. Equ. Appl. 22, 1483-1513 (2015) 
Shan Zhang

School of Mathematical Science

Nanjing Normal University

Nanjing 210023

China

e-mail: shanzhang86@163.com

Zuhan Liu

School of Mathematical Science Yangzhou University

Yangzhou 225002

China

e-mail: zhliu@yzu.edu.cn 